\title{
On-Chip Integratable Elastomeric Dome Valves for Glass Microfluidic Systems
}

\author{
Song-I Han, Youngdo Jung ${ }^{1}$, Ki-Ho Han* and Albert Bruno Frazier ${ }^{1}$ \\ School of Nano Engineering, Inje University, 607 Obang-dong, Gimhae, \\ Gyongnam 621-749, Republic of Korea \\ ${ }^{1}$ School of Electrical and Computer Engineering, Georgia Institute of Technology, \\ 777 Atlantic Drive, Atlanta, GA 30332-0250, USA
}

(Received July 2, 2007; accepted November 9, 2007)

Key words: microvalve, fluid-control device, microfluidic system, microfabrication, stereolithography

In this paper, we present a methodology for realizing integrated, pneumatically controlled dome valves for use with glass microfluidic systems. The dome valves were fabricated by attaching a microfluidic system interface (MSI), manufactured by stereolithography, to a glass microfluidic system. Both normally open and normally covered types of dome valves were designed to have a low dead volume of $12 \mathrm{nl}$. The results show that the normally open dome valve was closed at valve pressures greater than $150 \mathrm{kPa}$, and that the normally covered dome valve was opened when the fluid pressure was $7 \mathrm{kPa}$ higher than the valve pressure. Experimental results showed that the fluid pressure that resulted in leakage increased linearly with increasing valve actuation pressure from 0 to $450 \mathrm{kPa}$. We have demonstrated that the dome valves are practical fluid-control elements for use with glass microfluidic systems.

\section{Introduction}

For more than two decades, analytical microfluidic devices ${ }^{(1-3)}$ have been developed for manipulating and analyzing samples of biological and clinical interest. Microfluidic devices, when compared with macroscale devices, have advantages such as smaller geometrical size, shorter analysis times, less sample/reagent consumption, and disposability. Many researchers have successfully demonstrated singular functional microfluidic devices ${ }^{(4,5)}$ and transducers ${ }^{(6,7)}$ for fluid manipulation. However, the integration of analytical functions and multiple devices into a complex microfluidic system has shown modest development because of technical difficulties, such as the need for more complex packaging, the possibility of biological/chemical cross contamination

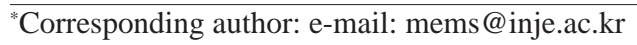


between functional compartments, and the possible need for different substrate materials to maintain compartment functionalities.

One practical method for solving some of the difficulties with fluid control in microsystems is to integrate microvalves into complex microfluidic systems. A number of microvalves have been developed from silicon and glass materials. ${ }^{(8)}$ Although such microvalves have been successfully demonstrated, most of them were realized using hybrid manufacturing methods resulting in relatively large dead volumes. To overcome the limitations of hybrid microvalves, elastomeric microvalves ${ }^{(6,9-12)}$ were designed and realized. These microvalves were fabricated using an elastomer substrate such as polydimethylsiloxane (PDMS), which PDMS can be easily used for fabricating the microfluidic devices including microvalves. Although PDMS-based microfluidic devices have been successfully demonstrated, the hydrophobicity and porosity of PDMS limits the types of chemical and biological assay applications that can be achieved without surface modification. ${ }^{(13,14)}$ In contrast, Borofloat glass-based microfluidic devices are better autofluorescent properties compare with plastic-based chips. ${ }^{(15)}$ In addition, glassbased microfluidic devices have shown advantages in applications such as solid-phase extraction (SPE), ${ }^{(16)}$ capillary electrophoresis (CE), ${ }^{(17)}$ and high-sensitivity fluorescence detection by CE. ${ }^{(18)}$ Since microfluidic devices ${ }^{(19)}$ fabricated by glass-to-glass thermal bonding technology provide a permanent bond, glass-based microfluidic devices are applicable for specialized applications that require high pneumatic pressure and high temperature (e.g., polymerase chain reaction (PCR) thermocycling). Since glass is a suitable material for various bioanalyses, the development of integrated fluid-control devices (e.g., microvalves) for application to glass-based microfluidic devices is critical.

In this paper, we present the design, fabrication, and characterization of normally open and normally covered dome valves that can be integrated with glass microfluidic devices on single chips. The dome-shaped microvalves have a low dead volume, show linearity between fluid leakage pressure and applied valve pressure, and have a large working range of valve pressures.

\section{Design and Fabrication}

\subsection{Design}

Figures 1(a)-1(d) show schematic views of the normally open and normally covered dome valves. The chemically etched valve hole was dome shaped to avoid fluid leakage at the edges of the mechanically drilled glass holes, as shown in Fig. 1(b). A flexible membrane, as an active element acting as a pneumatic valve, was forced down by the valve port when attaching the microfluidic system interface (MSI) ${ }^{(20)}$ on the glass chip. The membrane mechanically stops on the bottom glass, Fig. 1(c). The microchannel cross section beneath the microvalve was maintained without narrowing by the flexible membrane. The O-ring gasket in Fig. 1(b) was used to tightly hold the flexible membrane on to the glass chip, and to prevent leakage of the bonding adhesive into the dome-shaped valve hole when bonding the MSI to the glass chip. In recent reports $^{(4,6)}$ using elastomeric valve technologies on microfluidic devices, the flexible elastomeric membrane has been required to be incorporated into the fabrication process flow for the microfluidic device. The method reported herein decouples the microfluidic 


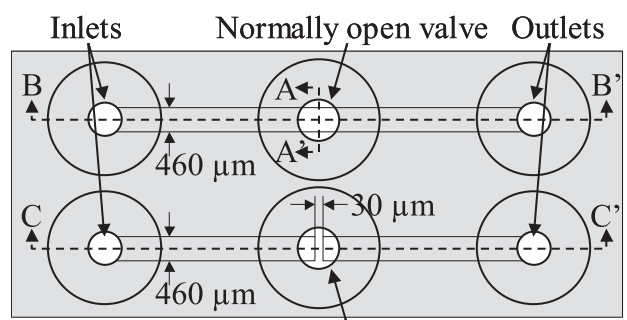

Normally closed valve

(a) Top view

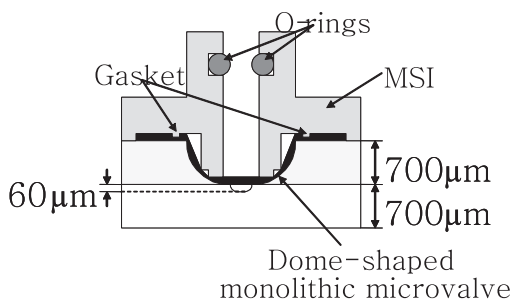

(b) A-A' cross-section view



(c) B-B' cross-section view of the normally open valve

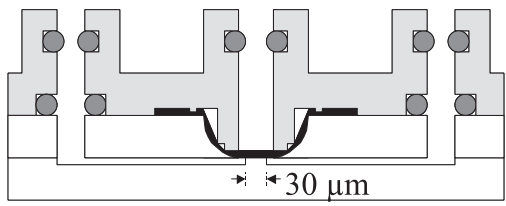

(d) C-C' cross-section view of the normally covered valve

Fig. 1. Schematic views of the normally open and normally covered dome-shaped microvalves.

glass chip fabrication process from that of the microvalve. The microvalve was created upon attaching the MSI on the glass chip. This decoupling allows the glass chip to be fabricated by conventional glass microfabrication technologies (e.g., glass-to-glass thermal bonding). Furthermore, microvalve technology can be implemented using various materials for the flexible membrane.

\subsection{Fabrication process}

Stereolithography (SLA) was used as the underlying fabrication technology for the MSI. The primary advantage of using SLA technology to fabricate the MSI was that it allowed the realization of arbitrary three-dimensional (3-D) structures with very few design constraints.(7) Furthermore, SLA allowed the integration of other component functionalities such as the incorporation of electrical, mechanical, and optical components as part of the fabrication process. The resulting MSI could easily be aligned on top of a complex microfluidic system using tooling holes or edges for alignment. Additionally, the use of O-rings as a means of achieving tight fluid seals eliminated the clogging of the microchannels with bonding adhesive upon attaching the MSI on the underlying microfluidic system. 
The glass chip was fabricated by conventional glass etching and bonding technologies. The bottom glass (Borofloat ${ }^{\top \mathrm{M}}$ glass, $0.7 \mathrm{~mm}$ thick, Howard Glass Co., Worchester, MA) was etched $60 \mu \mathrm{m}$ deep using 25\% HF solution. The dome-shaped valve holes (Fig. 2) were etched using 25\% HF solution for approximately 10 h until holes of the appropriate diameter were etched through the bottom of the top glass (Fig. 3(a)). A mechanical drill was used along with a SLA alignment jig to fabricate the through-holes in the glass used for fluid interconnections, as shown in Fig. 3(b). The top and bottom glasses were bonded together by glass-to glass thermal bonding at $685^{\circ} \mathrm{C}$ for $3.5 \mathrm{~h}$ (Fig. 3(c)).

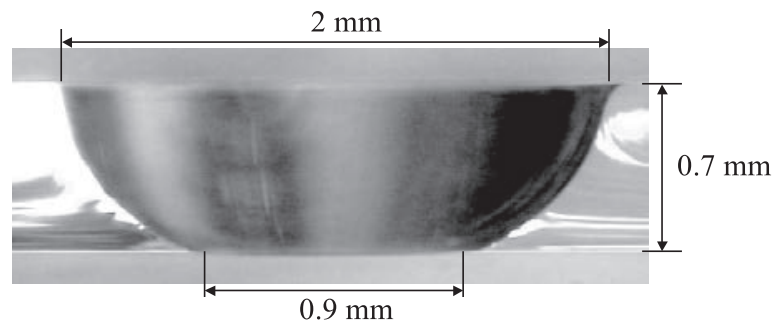

Fig. 2. Cross-section view of the dome-shaped valve hole formed by chemical wet etching.

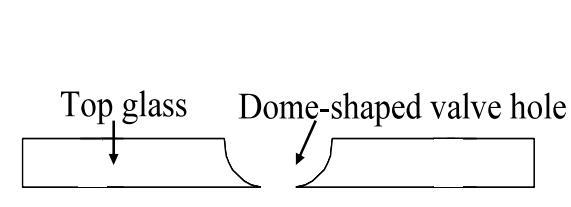

(a) Wet-etching valve hole in top glass
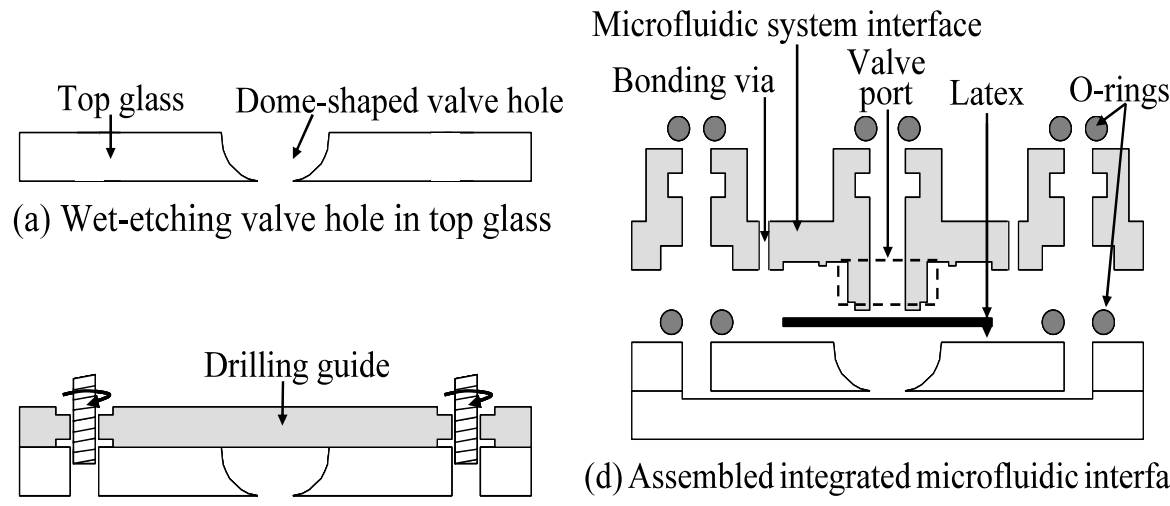

(b) Mechanical drilling through holes

(d) Assembled integrated microfluidic interface

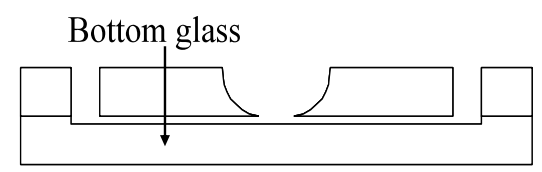

(c) Top glass thermally bonded



(e) MSI bonded to glass chip

\section{to bottom glass}

Fig. 3. Fabrication and assembly process used to realize a glass microfluidic device including microvalves. 
A nitrile rubber O-ring (Size 001-1/2, McMaster-Carr. Atlanta, GA) was used to avoid clogging of the microchannels with the low-viscosity UV adhesive (1187-M, DYMAX Co., USA) when bonding the MSI to the underlying glass chip. Another nitrile rubber O-ring (Size-001, McMaster-Carr) was used to provide a tight fluid seal between the external capillary tubing and the microfluidic interconnects. A $120-\mu \mathrm{m}$-thick latex membrane was used to form the active element of the microvalve (Fig. 3(d)). The MSI and the glass chip were aligned and placed in a clamped jig. Subsequently, the UV adhesive was placed into 'bonding' vias formed into the MSI, as shown in Fig. 3(e). Capillary force filled the UV adhesive into the gap between the MSI and the glass chip. The UV adhesive was then cured by placing it under a UV light for $30 \mathrm{~min}$, completing the fabrication of the MSI and glass chip, as shown in Fig. 3(e). Figure 4 shows the bottom and side views of the valve port including the gasket and air venting holes. The air venting holes were used to vent air in the gap between the MSI and the glass chip when filling the gab with UV adhesive. Figure 5 shows the fabricated glass-based microfluidic device before and after the bonding with the MSI.

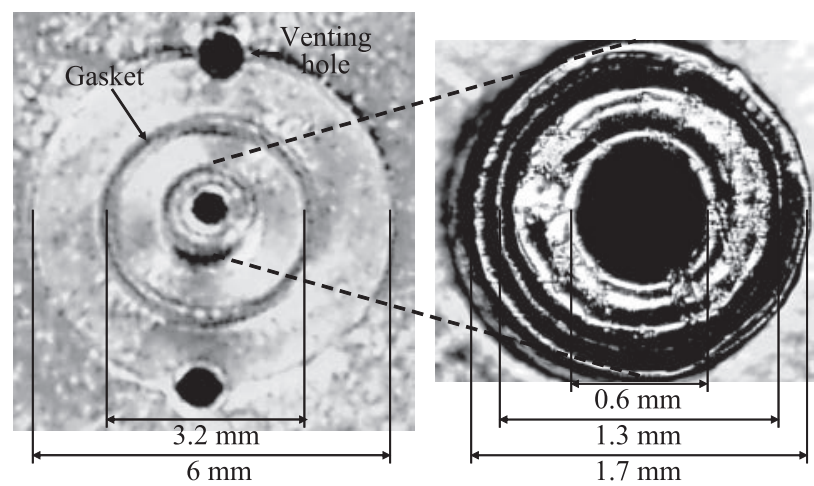

(a)

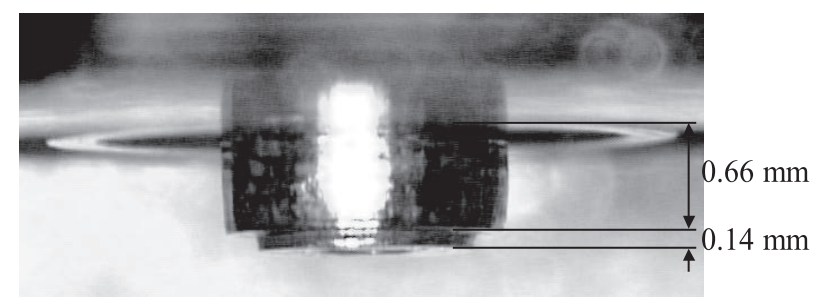

(b)

Fig. 4. Optical micrographs of the valve port fabricated by stereolithography. (a) Bottom view of the valve port, showing the gasket and air venting holes. (b) Side view of the valve port. 


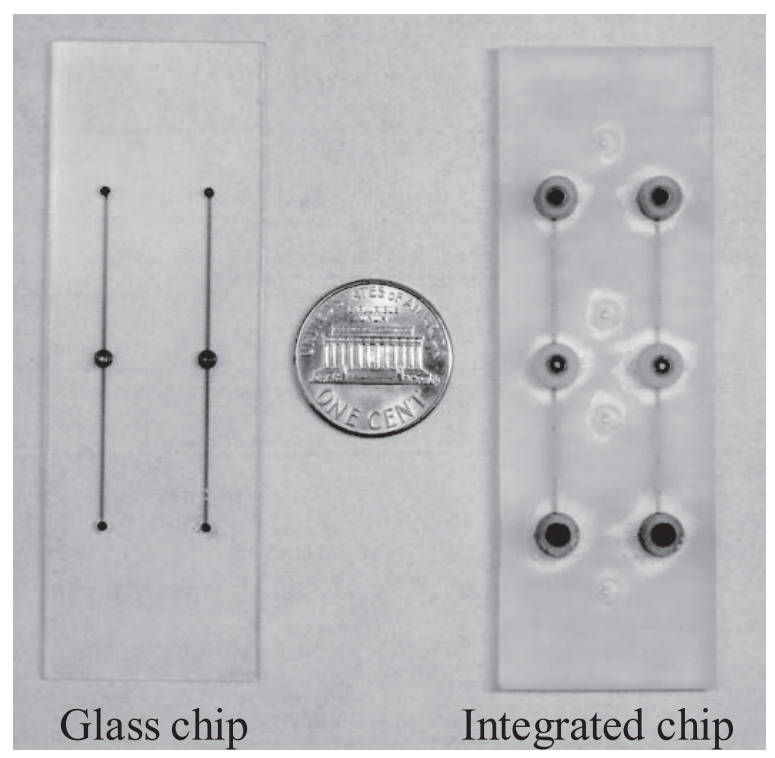

Fig. 5. Fabricated glass-based microfluidic device before and after bonding with the MSI. Red dye was used to clearly delineate the microchannels.

\section{Experimental Results}

Figure 6 shows the instrument setup used for characterizing the dome-shaped microvalves. As shown in Fig. 7, the blocked microchannel length in the closed state was $0.9 \mathrm{~mm}$, and the depth and width of the underlying microchannel were 60 and $400 \mu \mathrm{m}$, respectively. Therefore, the volume displaced by closing the normally open valve was calculated to be $12 \mathrm{nl}$. Figure 8 shows a graph of the fluid pressure required to cause leakage for a range of valve pressures for both the normally open and the normally covered dome valves. The experimental results show a linear relationship between these pressures in the instrument-limited range of valve pressures from 0 to $450 \mathrm{kPa}$. The normally open dome valve was closed when the valve pressure exceeded $150 \mathrm{kPa}$. A valve pressure of $150 \mathrm{kPa}$ was required for the $120 \mu \mathrm{m}$-thick latex membrane to completely seal the chemically etched microchannel. The normally covered dome valve was opened when the fluid pressure was $7 \mathrm{kPa}$ higher than the valve actuation pressure. Fluid pressures greater than $7 \mathrm{kPa}$ were required to overcome the elastic force of the latex membrane.

\section{Conclusions}

In this paper, we presented the design, fabrication, and characterization of dome valves for use with glass microfluidic systems. The dome-shaped microvalves, which 


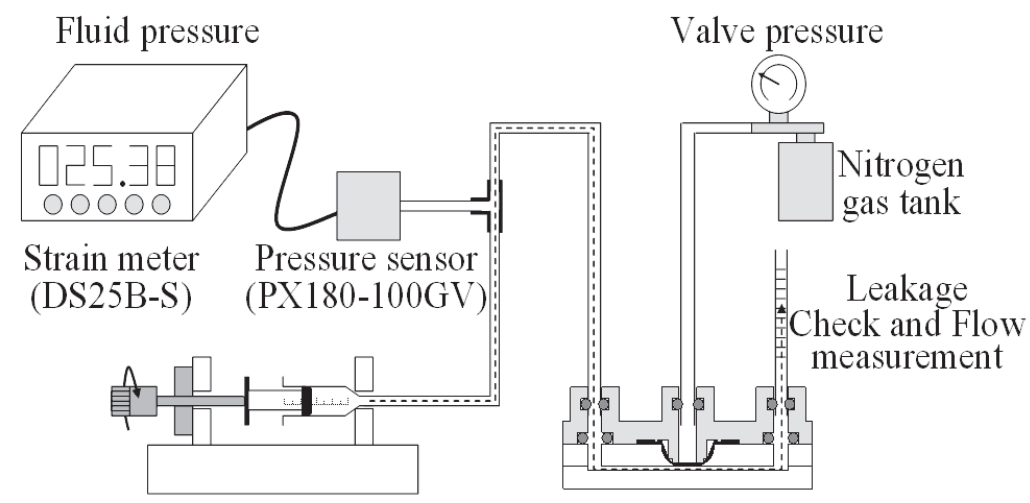

Fig. 6. Instrument setup for characterizing the fluid pressure required to cause leakage for a range of valve pressures.

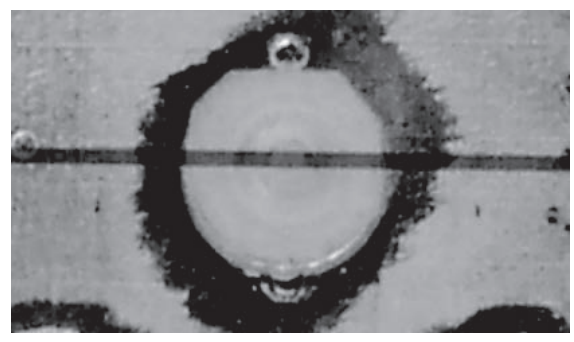

(a)

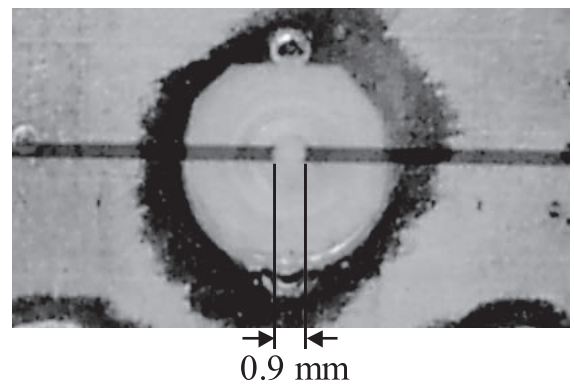

(b)

Fig. 7. Working states of the normally open dome valve. (a) Open state. (b) Closed state. 




Fig. 8. Graph showing fluid pressure required to cause leakage for a range of valve actuation pressures.

had a displaced channel volume of $12 \mathrm{nl}$ in the closed state, were actuated pneumatically in the range of valve pressures from 0 to $450 \mathrm{kPa}$. The dead volume presented herein is small compared with dead volumes of $35 \mathrm{nl}^{(6)}$ and $15.75 \mathrm{nl}^{(9)}$ reported by other elastomer microvalves. The dome-shaped valve has geometrical advantage to avoid the unwanted leakage, which cannot be inevitable for a microvalve fabricated by a straight hole. Additionally, it can be monolithically integrated on a microfluidic device, and the number of these can be especially built up at one time. The normally open dome valve was closed when the valve pressure exceeded $150 \mathrm{kPa}$. The normally covered dome valve was opened when the fluid pressure was $7 \mathrm{kPa}$ higher than the valve pressure. Consequently, the normally open and normally covered dome valves presented herein are reliable microfluidic control elements that enable the integration of analytical functionalities with glass microfluidic systems, and can be individually controlled according to the analytical functionalities and flow requirements of the microcompartments.

\section{Acknowledgement}

This work was supported by the 2007 Inje University research grant.

\section{References}

1 S. C. Terry, J. H. Jerman and J. B. Angel: IEEE Trans. Electron Devices ED-26 (1979) 1880.

2 O. Bakajin, T. A. J. Duke, J. Tegenfeldt, C.-F. Chou, S. S. Chan, R. H. Austin and E. C. Cox: Anal. Chem. 73 (2001) 6053.

3 Y. Huang, S. Joo, M. Duhon, M. Heller, B. Wallace and X. Xu: Anal. Chem. 74 (2002) 3362.

4 A. Y. Fu, C. Spence, A. Scherer, F. H. Arnold and S. R. Quake: Nat. Biotechnol. 17 (1999) 1109.

5 K.-H. Han and A. B. Frazier: Lab Chip 6 (2006) 265. 
6 W. H. Grover, A. M. Skelley, C. N. Lui, E. T. Lagally and R. A. Mathies: Sens. Actuators B 89 (2003) 315.

7 A. Han, O. Wang, M. Graff, S. K. Mohanty, T. L. Edwards, K.-H. Han and A. B. Frazier: Lab Chip 3 (2003) 150.

8 G. T. A. Kovacs: Micromachined Transducers Sourcebook (New York: McGraw-Hill, 1998) Chap. 9.

9 K. Hosokawa and R. Maeda: J. Micromech. Microeng. 10 (2000) 415.

10 Y.-C. Wang, M. H. Choi and J. Han: Anal. Chem. 76 (2004) 4426.

11 S. Lee, W. Jeong and D. J. Beebe: Lab Chip 3 (2003) 164.

12 T. Thorsen, S. J. Maerkl and S. R. Quake: Science 298 (2002) 580.

13 X. Ren, M. Bachman, C. E. Sims, G. P. Li and N. Allbritton: J. Chromatogr. B, Biomed. Sci. Appl. 762 (2001) 117.

14 S. Hu, X. Ren, M. Bachman, C. E. Sims, G. P. Li and N. Allbritton: Anal. Chem. 74 (2002) 4117.

15 A. Piruska, I. Nikcevic, S. H. Lee, C. Ahn, W. R. Heineman, P. A. Limbach, and C. J. Seliskar: Lab Chip 5 (2005) 1348.

16 K. A. Wolfe, M. C. Breadmore, J. P. Ferrance, M. E. Power, J. F. Conroy, P. M. Norris and J. P. Landers: Electrophoresis 23 (2002) 727.

17 D. J. Harrison, K. Fluri, K. Seiler, Z. Fan, C. S. Effenhauser and A. Manz: Science 261 (1993) 895.

18 B. C. Giordano, L. Jin, A. J. Couch, J. P. Ferrance and J. P. Landers: Anal. Chem. 76 (2004) 4705.

19 K.-H. Han and A. B. Frazier: J. Appl. Phys. 96 (2004) 5797.

20 K.-H. Han, R. D. McConnell, C. J. Easley, J. M. Bienvenue, J. P. Ferrance, J. P. Landers and A. B. Frazier: Sens. Actuators B 122 (2007) 337. 